\title{
Bio-inspired Aquatic Propulsion Mechanism Using a Fin with a Variable-Effective-Length Spring
}

- Development of a Propulsion Mechanism using the Swaying and Yawing Movements of a Fin-

\author{
Masataka Nakabayashi ${ }^{a^{*}}$, Hirotake Soyano ${ }^{b}$, Shunichi Kobayashi ${ }^{b^{*}}$, and Hirohisa Morikawa ${ }^{b}$ \\ ${ }^{a}$ Graduate School of Science and Technology, Shinshu University, Japan \\ ${ }^{b}$ Faculty of Textile Science and Technology, Shinshu University, Japan
}

\begin{abstract}
Elastic fins may represent a significant advance over traditional screw propellers for the propulsion of underwater vehicle. However, the optimum elasticity of the fin is not constant and changes with the required motion and the environment. To address this, we initially developed a fin with a variable-effective-length spring and documented the thrust characteristics given a simple yawing movement of the fin.
\end{abstract}

In this study, we developed a new propulsion mechanism that allows both swaying and yawing movements of the fin. We evaluated the behavior of the fin and the flow field surrounding the fin.

Index Terms - Propulsion mechanism, Variableeffective-length spring, Thrust force, Elastic fin, Flow visualization.

\section{INTRODUCTION}

The conventional screw propeller is widely used to propel ships and underwater vehicles. Recently, the use of a bio-inspired oscillating elastic fin has been proposed as an alternative propulsion mechanism. It is thought that the new mechanism will improve efficiency and safety, relative to the screw propeller. The proposed system resembles the caudal or pectoral fin of a fish. A number of studies have documented the development of this system for use in ships or underwater vehicles/robots [1-4]. Tangorra et al. [1] have initiated a research program to develop a maneuvering propulsor for unmanned undersea vehicles (UUVs). The propulsor is based on the pectoral fin of the bluegill sunfish. Recently, Nakashima et al. [2] developed a robotic dolphin that was propelled by a flexible fin using two springs. The authors also developed a simulation method to analyze the three-dimensional dynamics of the robot and conducted swimming experiments to evaluate the validity of the simulation method and the control algorithm. Nagai et al. [3] developed a robotic fish that uses a flexible fin with a coil spring. The authors described the effects of changing the spring constant on the oscillating frequency of the fin and the swimming number, which represents an ability of the animal's swimming relating with the beating frequency and the body length. Silas et al. [4] studied the mechanical properties of fin rays, which are a fundamental component of fish fin structure. The authors derived a linear elasticity model that predicts the shape of fin rays, given the input muscle actuation and external loading, and then tested the model using experiments that measured (i) the ray deflection for a given actuation at the muscular interface and, (ii) the force-displacement response under conditions of actuation and non-actuation.

The optimum elasticity of a fin is not constant and changes based on the behavior (e.g. swimming speed) and environment (e.g. uniform versus turbulent flow). However, it is very difficult to exchange fins of different stiffness while moving. To address this problem, a number of researchers have investigated the use of variable stiffness fins for the propulsion of aquatic vehicles. For example, Oda et al. [5] developed a variable-stiffness-spring for which the stiffness was changed by varying the spring coefficient to control the displacement of the spring. Morita et al. [6] evaluated the effect of changes in impedance on the joints of a robot by controlling mechanical softness and compliance. Matsuda et al. [7] developed a closed-loop multi-link robot and described the effect of controlling the distribution of stiffness in the joints on locomotion. Ishibashi et al. [8] developed a mass measurement system in microgravity using a variable-effective-length spring. Funakawa et al. [9] developed a variable stiffness mechanism for the joint region, and compared the loading of the experimental model with the dynamical model. Kawamura et al. [10] developed a mechanical element capable of variable stiffness by changing the pressure inside a laminated structure consisted of folded polypropylene sheets covered by vinyl sheet. Despite their promise, these mechanisms have not yet been applied to aquatic propulsion mechanisms.

We developed a variable-stiffness fin with a variable-effective-length spring. The apparent stiffness of the spring can be changed dynamically. We applied the concept to the development of a propulsion mechanism using a fin with the variable stiffness mechanism [11-12]. More recently, we described the thrust characteristics of the fin in the uniform flow velocity of water tunnel [13]. However, in these early models, the movement of the fin was limited to a simple yawing motion, which differs considerably from the movement of a caudal fin. Therefore, we developed a new propulsion mechanism using a fin that incorporates both swaying and yawing movements.

Our objective was to describe the behavior of the

* Corresponding author: Shinshu University, 3-15-1 Tokida, Ueda, Nagano 386-8567, Japan, E-mail: \{s07t108, shukoba\}@shinshuu.ac.jp 


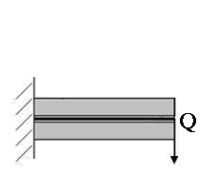

(a) Apparent Stiffness : Maximum $(H=0)$

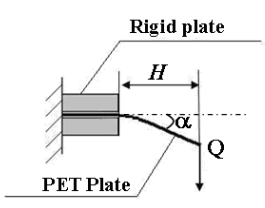

(b) Apparent Stiffness Middle $\left(0<H<L_{s}\right)$

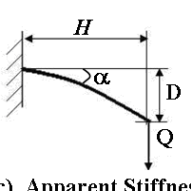

: Minimum $\left(H=L_{\mathrm{s}}\right)$

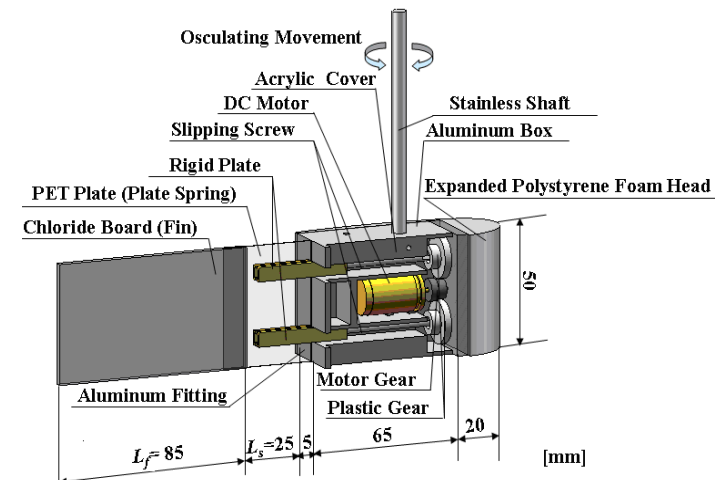

Fig. 2 Structure of a fin with a variable effective length spring

oscillating fin, the thrust force, and flow field around the fin.

\section{FIN WITH VARIABLE EFFECTIVE LENGTH SPRING}

\section{A. Principle of the Variable Effective Length Spring}

Figure 1 illustrates the principle of a variableeffective-length spring [11]. The effective length of the spring $(H)$ is changed by varying the length of rigid plates supporting a plate spring made of polyethylene terephthalate (PET). The length of the PET plate equals the maximum effective length of the spring $\left(L_{s}\right)$. The displacement $(D)$ and bending angle $(\alpha)$ increase with increasing $H$, assuming the tip of the spring is subject to a constant load. Thus, the apparent stiffness changes with an increase or decrease in $H$.

\section{B. Structure of a Fin with a Variable-Effective- Length Spring}

Figure 2 illustrates the structure of a fin with a variable-effective-length spring. The system consists of an aluminum box $(\mathrm{L} \times \mathrm{H} \times \mathrm{W}: \quad 65 \times 50 \times 30 \mathrm{~mm})$, an expanded polystyrene foam head, streamlined in shape, a fin consisting of a chloride plate (thickness $=3 \mathrm{~mm}$, length $L_{f}=85 \mathrm{~mm}$ ), and a variable-effective-length spring $\left(L_{s}=25 \mathrm{~mm}\right)$. A transparent acrylic plate cover is attached to the open side of the aluminum box to seal the fin. The rigid plates are reciprocated by a rotating DC motor. The effective length of the spring $(H)$ was changed by varying the supporting length of the rigid plates. The apparent stiffness of the fin changes with $H$.

\section{EXPERIMENTAL METHODS}

\section{A. Control and Measurement System}

Figures 3 and 4 show a photo of the propulsion mechanism and a schematic of the control and

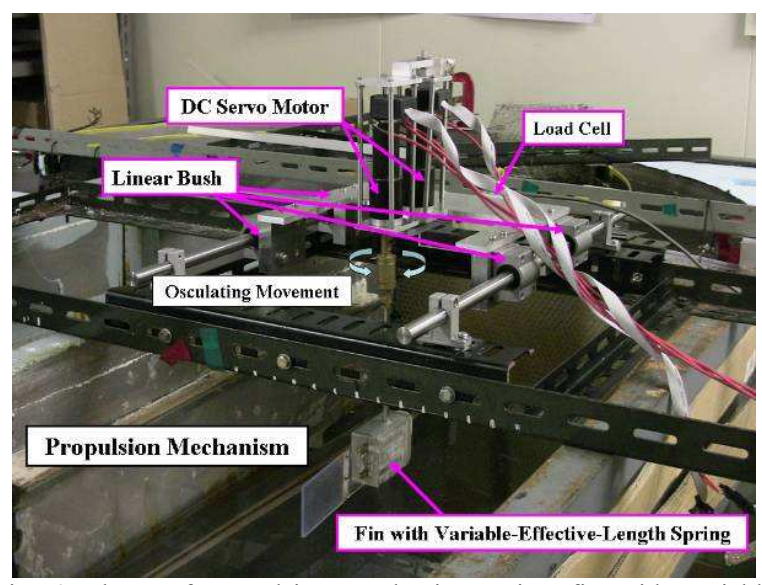

Fig. 3 Photo of propulsion mechanism using fin with variable effective length spring

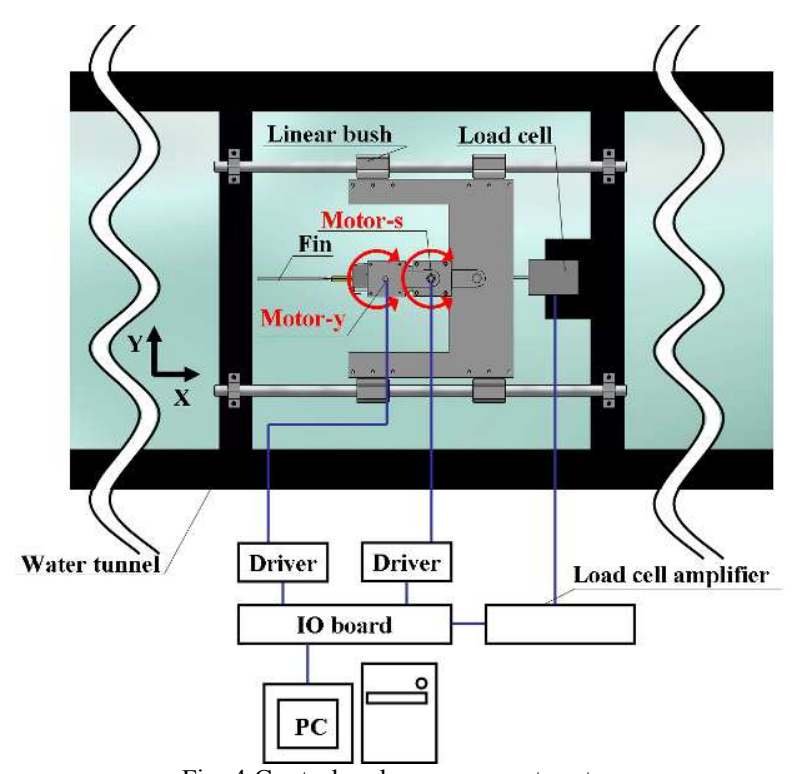

Fig. 4 Control and measurement system

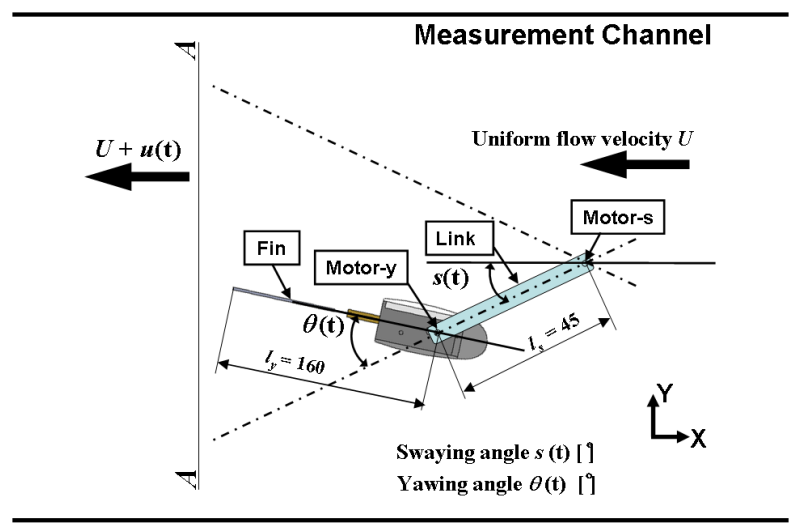

Fig. 5 Definition of swaying $s(t)$ and yawing $\theta(t)$ angles

measurement system, respectively. The propulsion mechanism was placed in the measurement channel of a water tunnel [13]. The measurement channel was $240 \mathrm{~mm}$ deep (water depth), $500 \mathrm{~mm}$ wide, and $800 \mathrm{~mm}$ long. The fin was positioned in the middle of the measurement channel (both horizontally and longitudinally). The uniform flow velocity $(U)$ could be changed over the range $U=0.05-0.20 \mathrm{~m} / \mathrm{s}( \pm 0.03 \mathrm{~m} / \mathrm{s})$ by varying the voltage supplying the underwater pump 
motors [13]. The $U$ was adjusted to set the average thrust force in one movement cycle $\left(F_{\text {avg }}\right)$ to zero. Under this scenario the thrust force generated by oscillating the fin equals the drag force from the uniform flow, corresponding to the self-propelled condition.

The yawing and swaying movements of the fin are controlled by the geared DC servomotors. The signal was transmitted from the PC to the driver, to control the speed of the motor. The swaying $s(t)$ and yawing $\theta(t)$ angles were defined as:

$$
\begin{aligned}
& s(t)=S \sin \omega t \\
& \theta(t)=\Theta \sin (\omega t-\phi) \\
& \omega=2 \pi / T
\end{aligned}
$$

where the $S$ is the amplitude of the swaying angle, $\Theta$ is the amplitude of the yawing angle, $\omega$ is the angular frequency of the oscillating fin, $\phi$ is the phase difference between swaying and yawing movements, $t$ is time, and $T$ is the oscillating cycle (Fig. 5). We tested the mechanism under the following conditions: $\Theta=30^{\circ}, S$ $=15^{\circ}, \omega=0.7,0.5$, and $0.4 \mathrm{~Hz}(T=1.5,2$, and $2.5 \mathrm{~s})$, and $\phi=90^{\circ}$. The propulsion mechanism was supported by a linear guide and was able to move along the $\mathrm{x}$ direction axis (shown in Fig.5). The mechanism was connected to a load cell to enable measurement of the thrust force $(F)$. In addition, we attached four fluorescent markers $(3 \times 3 \mathrm{~mm})$ to the underside of the fin (Fig.6) to visualize the oscillating behavior of the fin. We used a digital video camera (frame rate: 30 frame/s, image: $740 \times 480$ pixels) to record images of the markers. The images were analyzed using twodimensional motion analysis software.

The average thrust force $F_{\text {avg }}$ was equal to zero for all scenarios. Then, we evaluated the relativity between the changes in thrust force $(F)$ and the oscillating movement of the fin needed to satisfy the requirement that $U$ corresponds to the self-propellant speed.

\section{B. Method of Flow Visualization}

Figure 7 shows a schematic diagram of the method of flow visualization. We used a tracer (diaion particles: average particle size, $250 \mu \mathrm{m}$, S.G. 1.02) to visualize flow in the measurement channel. The fin was illuminated on both sides with light sheets as follows.

We used two HID-lamps (luminous flux: 5,400 lm). One was fixed to the side of the measurement water channel and the other was fixed to the bottom of the channel. The white light from the HID-lamp passed through a slit (thickness: $5 \mathrm{~mm}$ ) to form a sheet of light. The light sheet originating from the lamp mounted below the channel illuminated the side of the fin after being reflected with a mirror fixed to the inside of the water tunnel. The second HID lamp created a light sheet which illuminated the other side of the fin through a slit in the side of the water tunnel. The remainder of the channel was covered with black sheets to block extraneous light. We used a high speed video camera (frame rate: 120 frame/s, image: $740 \times 480$ pixels) to

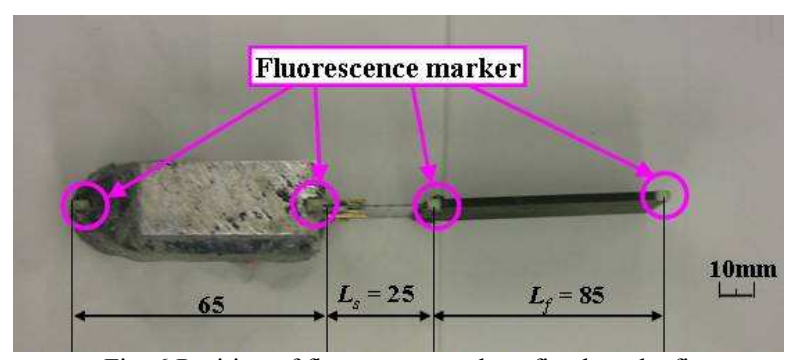

Fig. 6 Position of fluorescent markers fixed on the fin

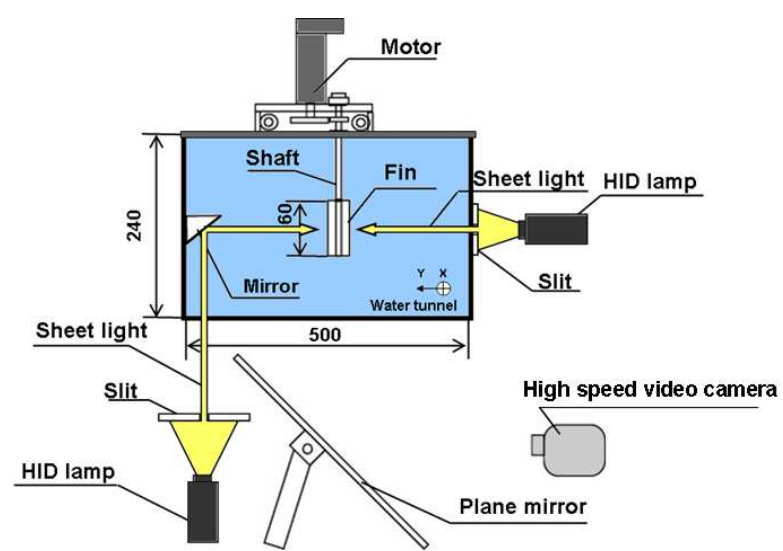

Fig. 7 Schematic diagram of the flow visualization device

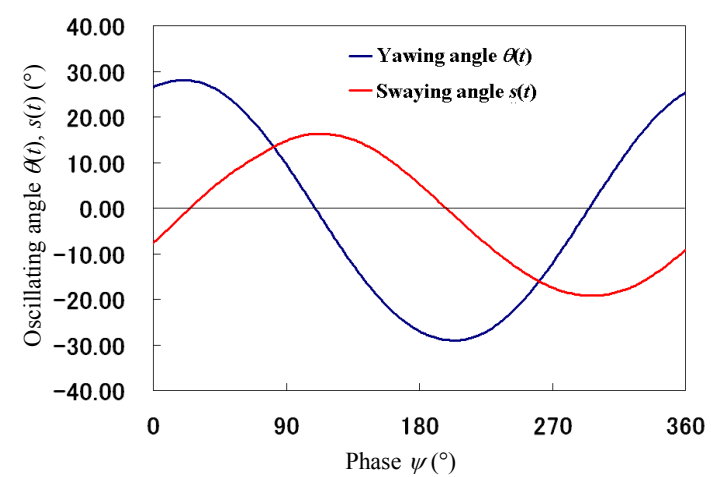

Fig. 8 Changes in the swaying $s(t)$ and the yawing $\theta(t)$ angles in one oscillating cycle in a uniform flow environment $\left(T=2 \mathrm{~s}, \Theta=30^{\circ}, S=15^{\circ}, F_{\text {avg }}=0 \mathrm{~N}\right)$

capture images of the flow. The camera was mounted below the measurement channel to prevent inflection caused by the fluid level. For the analysis of the flow field, we used a particle image velocimetry (PIV) system.

\section{RESULTS AND DISCUSSION}

\section{A. Behavior of the Fin during Yawing and Swaying Movements in a Uniform Flow Environment}

Changes in the swaying $s(t)$ and yawing $\theta(t)$ angles of the fin are shown in Fig. 8. These angles were measured by a potentiometer geared to each servo motor. The phase difference $(\phi)$ between $s(t)$ and $\theta(t)$ was set at $90^{\circ}$. The results of the two-dimensional motion analysis describing the behavior of the oscillating fin during yawing and swaying movements are shown in Fig. 9. Here, $F_{\text {avg }}=0$ in one oscillating cycle in uniform flow. Under this scenario the thrust 


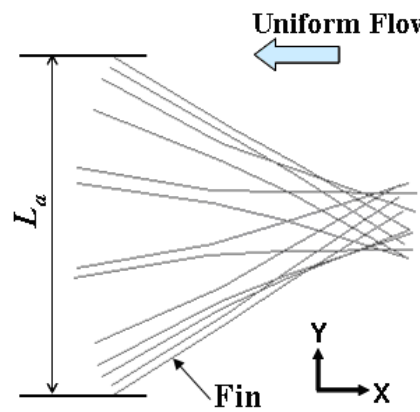

(a) Maximum apparent stiffness $(H=0, U=70.1 \mathrm{~mm} / \mathrm{s})$

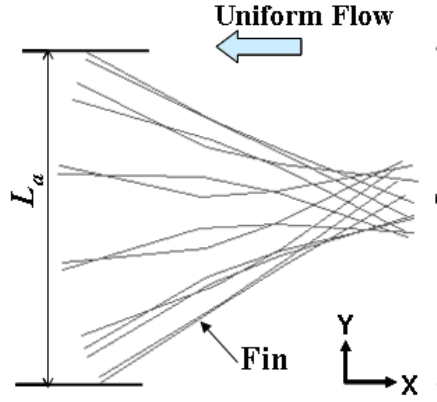

(b) Middle apparent stiffness $(H=3 L / 5, U=85.6 \mathrm{~mm} / \mathrm{s})$

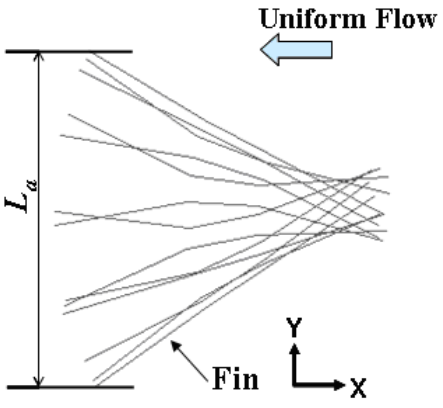

(c) Minimum apparent stiffness $\left(H=L_{s}, U=75.1 \mathrm{~mm} / \mathrm{s}\right)$

Fig. 9 Behavior of the oscillating fin in a uniform flow environment $\left(T=2 \mathrm{~s}, \Theta=30^{\circ}, S=15^{\circ}, F_{\text {avg }}=0 \mathrm{~N}\right)$

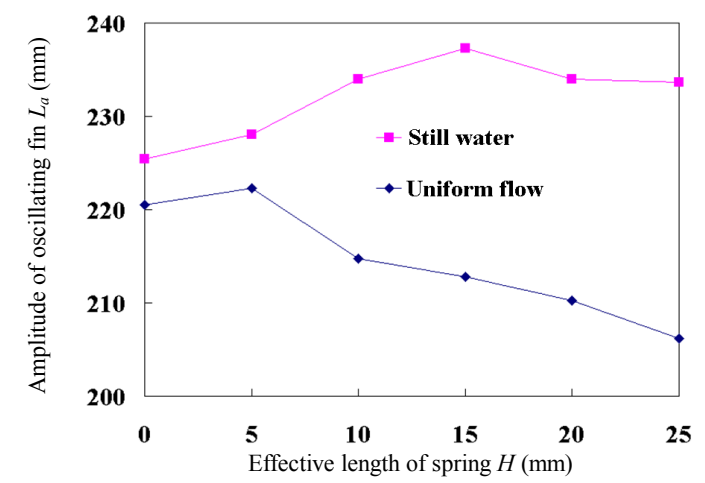

Fig. 10 Relationship between the amplitude of the oscillating fin $L_{a}$ and effective length of the spring $H\left(T=2 \mathrm{~s}, \Theta=30^{\circ}, S=15^{\circ}\right)$

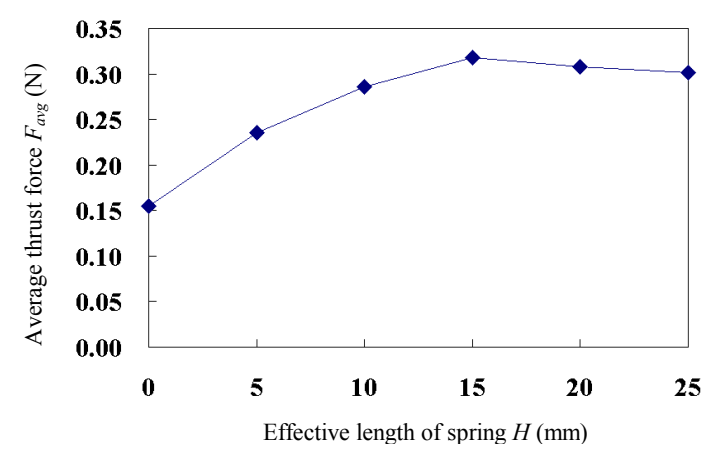

Fig. 11 Relationship between the average thrust force $F_{\text {avg }}$ and the effective length of the spring $H$ in still water $\left(T=2 \mathrm{~s}, \Theta=30^{\circ}, S=\right.$ $\left.15^{\circ}\right)$

force of the propulsion mechanism is balanced. The behaviors of the oscillating fin when the effective length of the spring $H$ is maximum, mid, and minimum are depicted in panels a, b, and c of Fig. 9, respectively. The bending angle of the fin $(\alpha)$ increased as $H$ increased because the apparent stiffness decreases as $H$ increases.

The relationship between the amplitude of the oscillating fin $\left(L_{a}\right)$ and $H$ in uniform flow and still water is shown in Figure 10. In this graph, the uniform flow velocity at each setting of $H$ is described in Fig. 12. The amplitude $L_{a}$ in still water increased with an increase in the $H$, peaking when $H=15 \mathrm{~mm}$ and tending to decrease thereafter. The increase in $L_{a}$ was associated with increasing $\alpha$ and a decrease in the apparent stiffness of the fin. After peaking, $L_{a}$ decreased because $\alpha$ was too large. Furthermore, $L_{a}$ was small in the uniform flow model relative to the still water model because the fin was subject to the drag force associated with flow. Thus, the tendency for $L_{a}$ to decrease with an increase in $H$ could be attributed to an increase in the drag force caused by a reduction in the apparent stiffness of the fin.

\section{B. Relationship between Average Thrust Force $F_{\text {avg }}$ and $H$ in Still Water.}

Figure 11 shows the relationship between average thrust force $\left(F_{\text {avg }}\right)$ and the effective length of the spring $(H)$ in still water. $F_{\text {avg }}$ increased with increasing $H$, peaking when $H=15 \mathrm{~mm}$, and decreasing thereafter. This pattern was similar to described for $L_{a}$ in still water. These results presented that $F_{a v g}$ varies with changes in $L_{a}$ because the fluid force generated by the oscillating fin changed with $L_{a}$. When $T$ was decreased to $1.5 \mathrm{~s}, F_{\text {avg }}=0.52 \mathrm{~N}$ and $L_{a}=231.3 \mathrm{~mm}$ for $H=20$ $\mathrm{mm}$ and $F_{\text {avg }}=0.41 \mathrm{~N}$ and $L_{a}=226.1 \mathrm{~mm}$ for $H=25$ $\mathrm{mm}$. Thus, $F_{\text {avg }}$ may also be increased by increasing $L_{a}$ when $T$ is varied in still water.

\section{Relationship between Uniform Flow Velocity (U), equivalent to the self-propelled speed, and the Effective Length of the Spring $(H)$}

We estimated the optimal effective length of the spring $\left(H_{u-\max }\right)$ needed to generate the maximum uniform flow velocity corresponding to the selfpropelled speed. Fig. 12 shows the relationship between $H$ and $U$ when $F_{\text {avg }}=0$ in one oscillating cycle. The drag force of the uniform flow that the fin received was decreased by reducing the apparent stiffness of the fin by increasing $H$. However, the fluid force generated by oscillating the fin decreased the bending angle of the fin by reducing the apparent stiffness of the fin. Therefore, $U$ increased as $H$ increased, although there was a point after which $U$ tended to decrease. Thus, the optimal apparent stiffness was defined as the apparent stiffness of the fin required to generate the maximum $U$.

The $H_{u-\max }$ needed to generate the maximum $U$ tended to increase with a decrease in the oscillating cycle $(T)$. When $T=1.5 \mathrm{~s}, U=98.0 \mathrm{~mm} / \mathrm{s}$ and $L_{a}=$ $194.9 \mathrm{~mm}$ for $H=20 \mathrm{~mm}$ and $U=96.0 \mathrm{~mm} / \mathrm{s}$ and $L_{a}=$ $187.1 \mathrm{~mm}$ for $H=25 \mathrm{~mm}$. Thus, we estimate that the fluid force generated by oscillating the fin when $H=20$ 


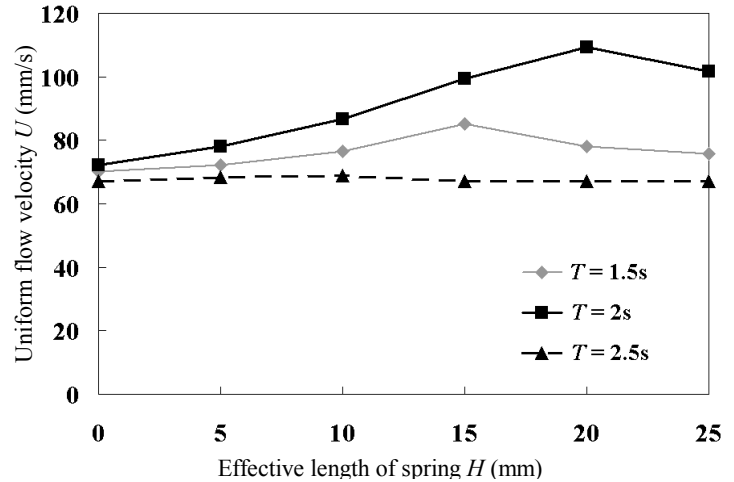

Fig. 12 Relationship between uniform flow velocity $U$ and effective length of the spring $H\left(T=2 \mathrm{~s}, \Theta=30^{\circ}, S=15^{\circ}, F_{\text {avg }}=0 \mathrm{~N}\right)$

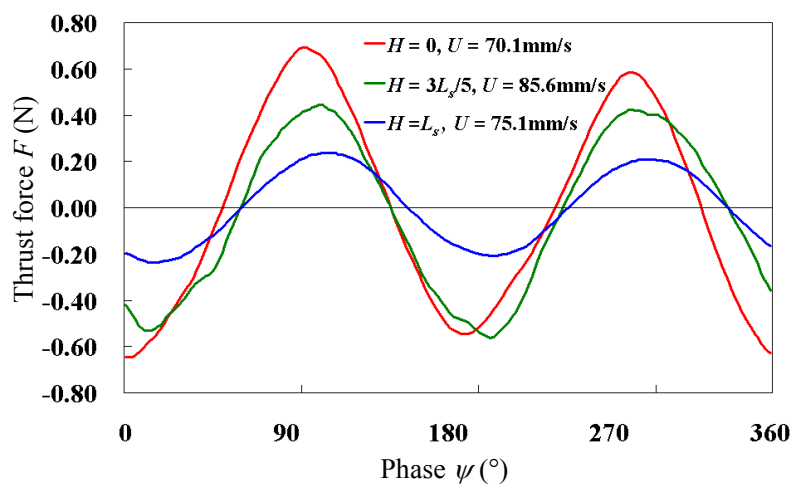

Fig. 13 Variation of thrust force $F$ in a uniform flow environment $\left(T=2 \mathrm{~s}, \Theta=30^{\circ}, S=15^{\circ}, F_{\text {avg }}=0 \mathrm{~N}\right)$
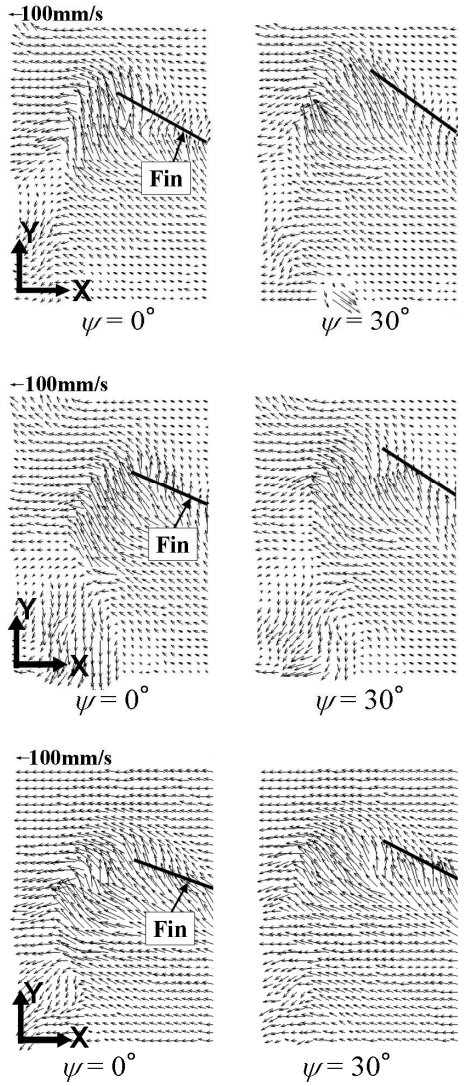

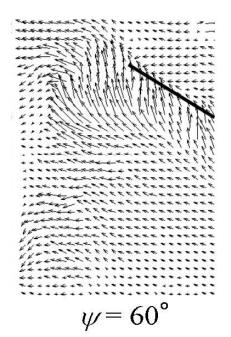

(a) Maximum apparent stiffness $(H=0, U=70.1 \mathrm{~mm} / \mathrm{s})$
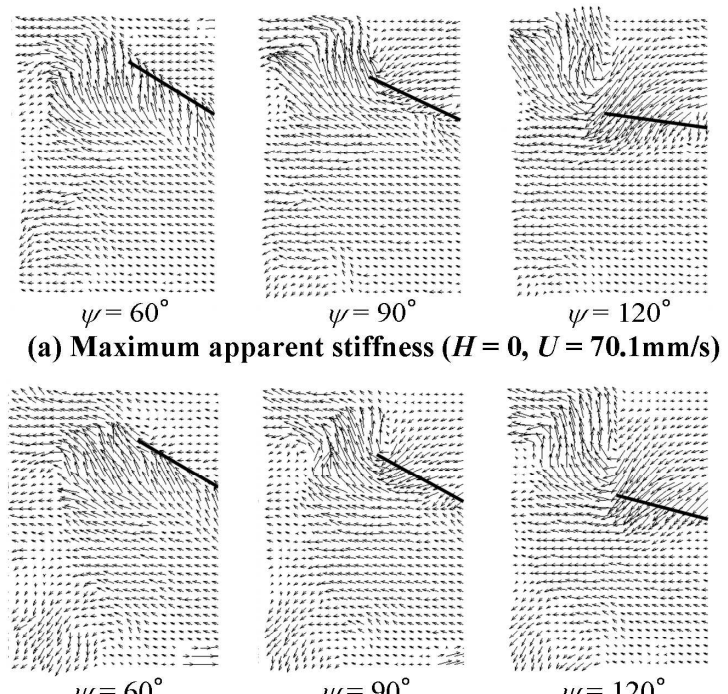

$\psi=120^{\circ}$

(b) Minimum apparent stiffness $\left(H=L_{s}, U=75.1 \mathrm{~mm} / \mathrm{s}\right)$

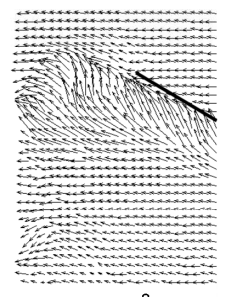

$\psi=60^{\circ}$

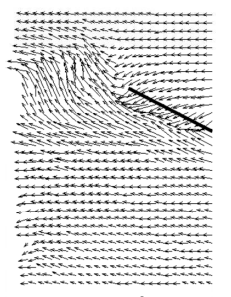

$\psi=90^{\circ}$

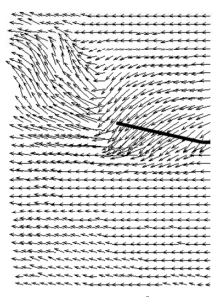

$\psi=120^{\circ}$
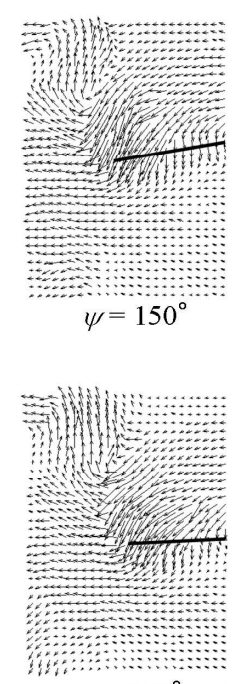

$\psi=150^{\circ}$

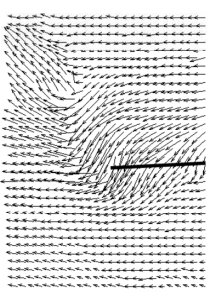

$\psi=150^{\circ}$
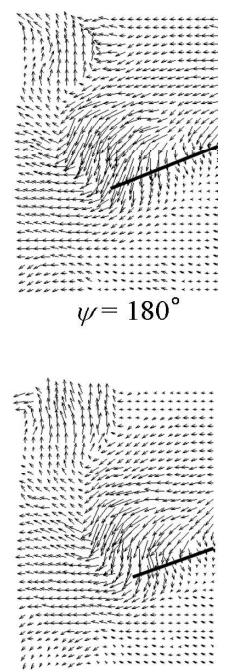

$\psi=180^{\circ}$

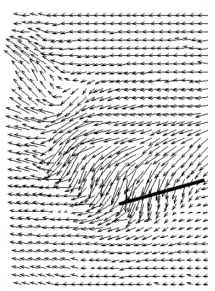

$\psi=180^{\circ}$

(c) Optimal apparent stiffness $\left(H=3 L_{s} / 5, U=85.6 \mathrm{~mm} / \mathrm{s}\right)$

Fig. 14 Flow velocity vector around the fin in a uniform flow environment $\left(T=2 \mathrm{~s}, \Theta=30^{\circ}, S=15^{\circ}, F_{\text {avg }}=0 \mathrm{~N}\right)$

$\mathrm{mm}$ is greater than when $H=25 \mathrm{~mm}$. This is also true when $T=2.0 \mathrm{~s}$.

\section{Variation of Thrust Force $(F)$ during Yawing and Swaying Movements in a Uniform Flow Environment}

The thrust force $(F)$ was measured in a uniform flow environment ( $U$ equivalent to the self-propelled speed) where $F_{\text {avg }}=0$. The variation in the thrust force when $\Theta=$ $30^{\circ}$ and $S=15^{\circ}$ in one oscillating movement cycle is shown in Figure 13. Our results demonstrate that the amplitude of the thrust force decreases as $H$ increases.

This change is associated with a decrease in the drag force of the fin while moving, caused by a reduction in $L_{a}$.
The thrust force changed rapidly between positive and negative when the apparent stiffness of the fin was maximized during the oscillating movement. Conversely, when the apparent stiffness of the fin was at a minimum, the thrust force changed very little. Thus, the dependence of propulsion speed on the thrust force was small. Given this, we estimated that the highest flow velocity corresponding to the self-propelled speed was generated when the thrust force was stable.

\section{E. Visualization of the Flow around the Fin}

The flow velocity vectors surrounding the fin are shown in Figure 14. The velocity vectors depict the uniform flow velocity and the disturbance to uniform flow induced by the oscillating movement of the fin. Panel (a) 

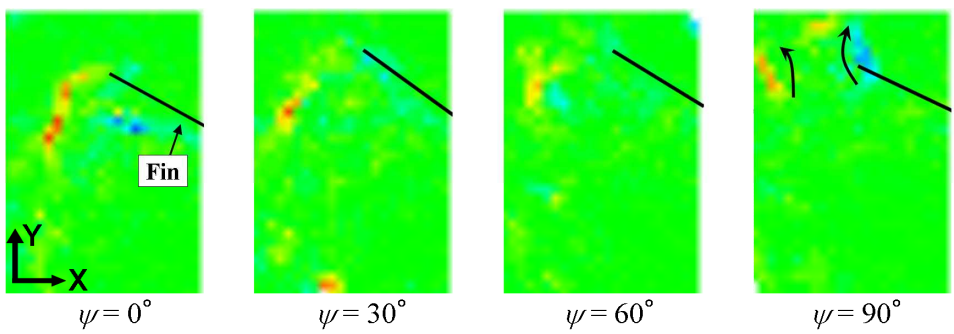

(a) Maximum apparent stiffness $(H=0, U=70.1 \mathrm{~mm} / \mathrm{s})$
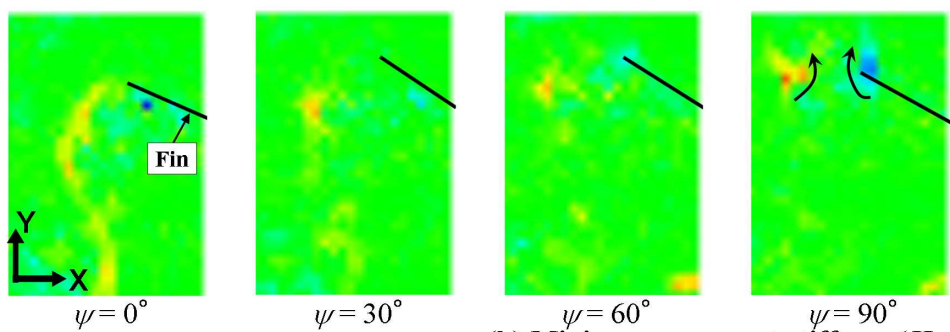
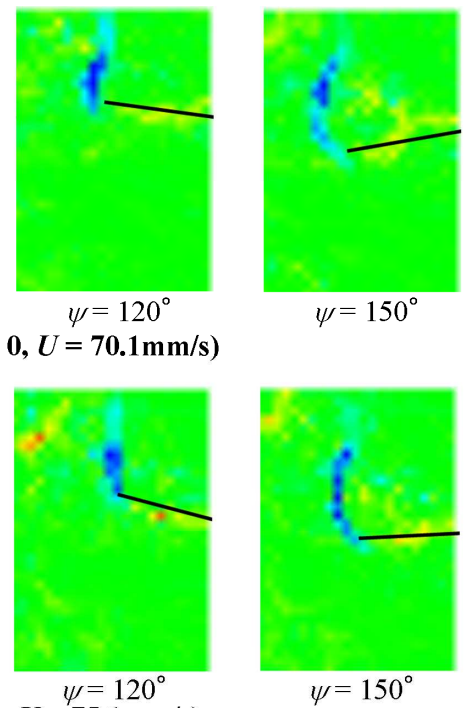

$\psi=150^{\circ}$

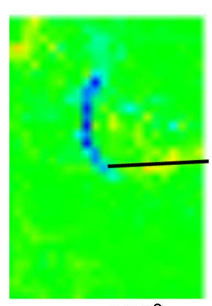

$\psi=150^{\circ}$

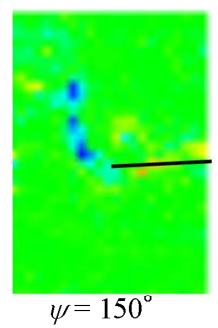

$\psi=120$

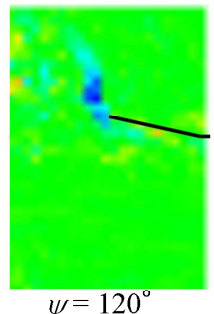

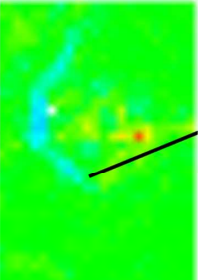

$\psi=180^{\circ}$

(b) Minimum apparent stiffness $\left(H=L_{s}, U=75.1 \mathrm{~mm} / \mathrm{s}\right)$
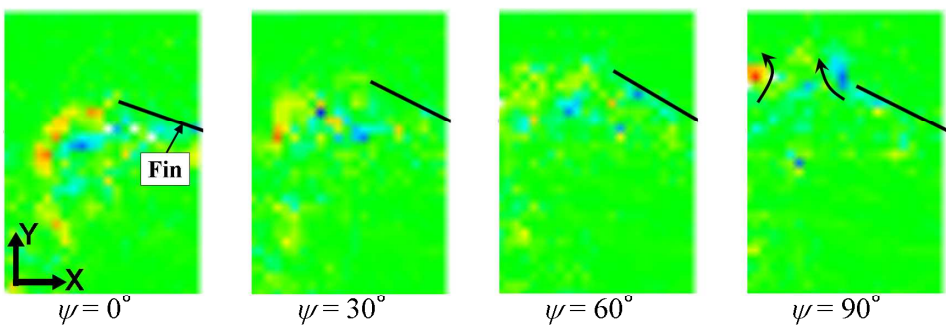

(c) Optimal apparent stiffness $\left(H=3 L_{s} / 5, U=85.6 \mathrm{~mm} / \mathrm{s}\right)$

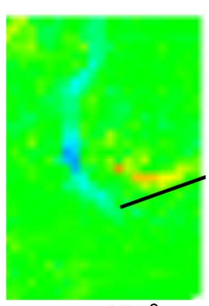

$\psi=180^{\circ}$
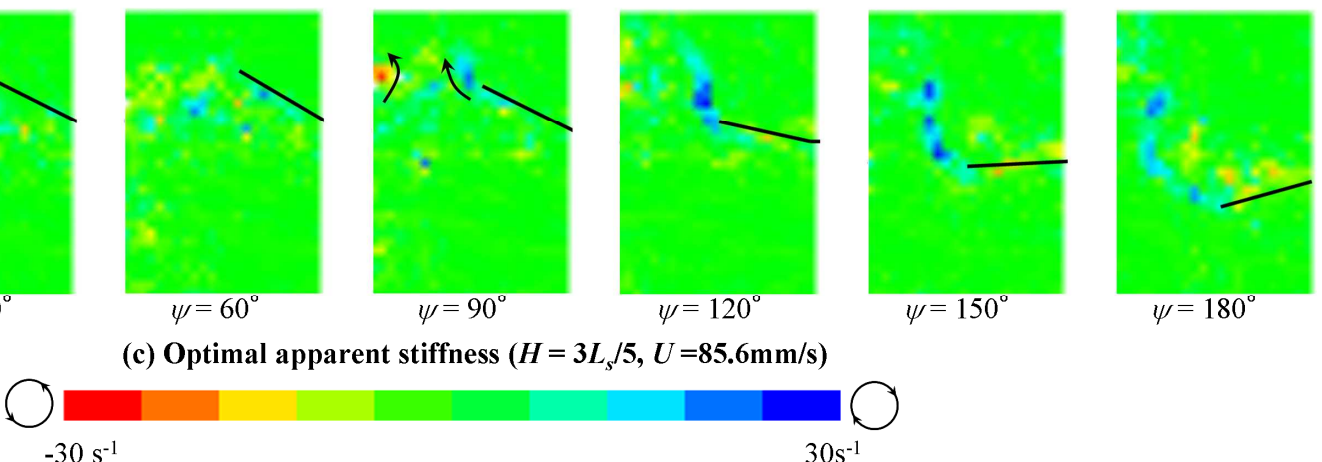

$30 \mathrm{~s}^{-1}$

Fig. 15 Distribution of vortices around the fin in a uniform flow environment $\left(T=2 \mathrm{~s}, \Theta=30^{\circ}, S=15^{\circ}, F_{\text {avg }}=0 \mathrm{~N}\right)$

illustrates the outcome when $H=0$, corresponding to illustrates the effect of minimizing apparent stiffness $(H=$ $L_{s}$ ). In both instances, the flow velocity vectors in the lateral direction are large. Thus, we conclude that the lateral force of the propulsion mechanism is also high under these conditions. The maximum $U$ (in Fig.14 (c)) occurs when $H=3 L_{s} / 5$ and is associated with the orientation of the flow velocity vector in the backward propellant direction.

We also recorded the vorticity around the fin during the oscillating movements. Figure 15 illustrates the distribution of vortices around the fin in a uniform flow environment. When $\psi=90^{\circ}$ (maximum thrust force), a pair of vortex rings were generated at the edge of the fin for all values of $H$. Arrows between the pairs of counter rotating vortices in the figure show the direction of the fluid jet in each scenario. The direction of the fluid jet under optimal stiffness conditions $\left(H_{u-\max }=3 L_{s} / 5\right)$ was closer to the backward direction of the propulsion mechanism (Fig. 4, x-direction) than in other scenarios. The fluid momentum that contributes to propulsion under optimal conditions was estimated to be large when compared with the other two scenarios $(H=0$ and $H=$ $\left.L_{s}\right)$.

\section{CONCLUSIONS}

We developed a fluid propulsion mechanism using a fin that has a variable effective length spring allowing both swaying and yawing movements. The behaviors of the fin, the thrust characteristics, and flow field around the fin in one oscillating movement were discussed. The following results were obtained:

(1) The bending angle of oscillating the fin $(\alpha)$ is increased by increasing the effective length of the spring $(H)$.

(2) The amplitude of the oscillating fin $\left(L_{a}\right)$ and the average thrust force $\left(F_{\text {avg }}\right)$ in still water increased to a point with an increase in $H$, after which the amplitude tended to decrease.

(3) The $H_{u-\max }$ required to generate the maximum $U$ increased with a decrease in the oscillating cycle $T$.

(4) The amplitude of the thrust force decreased with increasing $H$ in a uniform flow environment.

(5) The direction of the flow velocity vector is closer to the backward propellant direction when $H=3 L_{s} / 5$ than when $H=0$ or $H=L_{s}$.

An evaluation of the thrust characteristics under a number of scenarios (e.g. when $T$ is smaller or $\Theta$ and $S$ are larger) and in a dynamic state (changing $H$ during the oscillating movement of the fin) is needed to further advance our understanding of the utility of this approach to propelling underwater vehicle.

\section{ACKNOWLEDGMENTS}

This study was supported by a research grant from the Global COE program. 


\section{REFERENCES}

[1] Tangorra, J.L., Davidson, S.N., Hunter I.W., Madden, P.G.A., Lauder, G.V., Dong, H., Bozkurttas, M., and Mittal, R., The Development of a Biologically Inspired Propulsor for Unmanned Underwater Vehicles, IEEE Journal of Oceanic Engineering, Vol. 32, No. 3, (2007), pp.533-550.

[2] Nakashima, M., Tsubaki, T., and Ono, K., Three-Dimensional Movement in Water of the Dolphin Robot (2nd Report, Control Between Two Posture by Combination Motion of Roll and Pitch), Transactions of the Japan Society of Mechanical Engineering, Series C, Vol.71, No.702 (2005), pp.307-314.

[3] Nagai, M. and Tanata, I., Hydrodynamics of Drag and propulsion (Learn for Ability of aquatic organism) (in Japanese), Ship \& Ocean Foundation (1996), pp.187-195.

[4] Alben, S., Madden, P.G.A., and Lauder, G.V., The mechanics of active fin-shape control in ray-finned fishes, Journal of the Royal Society, Interface, (2007), pp.243-256

[5] Oda, J., Wong, A., and Matsumoto, N., Trial Formation of Variable-Stiffness Spring and Its Application to Displacement Control Problems, Transactions of the Japan Society of Mechanical Engineers, Series C, Vol. 59, No. 564 (1993), pp.262-267.

[6] Morita, T., and Sugano, S., Mechanical Softness and Compliance Adjustment, Journal of the Robotics Society of Japan, Vol. 17, No. 6 (1999), pp.790-794.

[7] Matsuda, M., Locomotion of Closed Loop Robot Using Stiffness Distribution Control, Transaction of the Robotics Society of Japan, Vol. 25, No. 2 (2007), pp.231-240.

[8] Ishibashi, R., Ozawa, R., and Kawamura, S., Development of
Mass Measurement System in Microgravity with a Variable Stiffness Mechanism, Transactions of the Society of Instrument and Control Engineers, Vol.43, No. 11, (2007), pp.1007-1014.

[9] Funakawa, Y., Konno, A., and Uchiyama, M., Joint Mechanism with Variable Stiffness, Transactions of the Japan Society of Mechanical Engineering, Series C, Vol.74, No. 744 (2008), pp.115 121.

[10] Kawamura, S., Yamamoto, T., Ishida, D., Nakayama, Y., and Tabata, O., Development of Wearable Elements with Variable Stiffness, Transactions of the Japan Society of Mechanical Engineering, Series B, Vol.69, No. 688 (2003), pp.182-187.

[11] Kobayashi, S., Nakabayashi, M., and Morikawa, H., Bioinspired Propulsion Mechanism in Fluid Using Fin with Dynamic Variable-Effective-Length Spring, Journal of Biomechanical Science and Engineering, Vol.1, No. 1 (2006), pp.280-287.

[12] Nakabayashi, M., Kobayashi, R., Kobayashi, S., and Morikawa, H., Propulsion Mechanism in Fluid Using Fin with Dynamic Variable-Effective-Length Spring (Evaluation of Thrust Efficiency and Flow-Visualization around the Fin), Transactions of the Japan Society of Mechanical Engineering, Series B, Vol.73, No. 736 (2007), pp.115-123.

[13] Nakabayashi, M., Kobayashi, R., Kobayashi, S., and Morikawa, H., Bioinspired Propulsion Mechanism Using a Fin with a Dynamic Variable-effective-Length Spring (Evaluation of Thrust Characteristics and Flow around a Fin in Uniform Flow), Journal of Biomechanical Science and Engineering, Vol.4, No.1, (2009), pp.82-93. 